\title{
One type of duplex appendix: horseshoe appendix
}

This article was published in the following Dove Press journal:

Therapeutics and Clinical Risk Management

\author{
Jin Liu',* \\ Chengyong Dong',* \\ Haibo Wang ${ }^{2}$ \\ Deguang Sun ${ }^{2}$ \\ Rui Liang ${ }^{2}$ \\ Zhenming $\mathrm{Gao}^{2}$ \\ Liming Wang ${ }^{1,2}$
}

'General Surgery, Center for New Material and New Precision

Technology of Cancer Therapy, Dalian Medical University, Dalian, Liaoning,

China; ${ }^{2}$ Division of Hepatobiliary and Pancreatic Surgery, Department of General Surgery, The Second Affiliated Hospital of Dalian Medical University, Dalian, Liaoning, China

*These authors contributed equally to this work
Correspondence: Liming Wang; Haibo Wang

Division of Hepatobiliary and Pancreatic Surgery, Department of General Surgery, The Second Affiliated Hospital of Dalian Medical University, No 467, Zhongshan Road, Shahekou District, Dalian, Liaoning II 6023, China

Tel/fax +86 4II 8467 I29I

Email wangbcc259@I63.com; whbsw2005@126.com

\begin{abstract}
A horseshoe appendix is a subtype of duplex appendix, in which the appendix is shaped like a circle that may lead to an internal hernia and result in intestinal necrosis. This subtype is extremely rare, with only 13 cases reported worldwide to date, and easily triggers a series of medico-legal consequences due to the neglect of another infection base of the appendix. We describe a 22-year-old man who presented with a 3-day history of fever and was diagnosed with pneumonia. After receiving antibiotics for 3 days in the Department of Pneumology, he was found to have a periappendiceal abscess. He underwent appendectomy after 3 days of conservative treatment failure in the Department of General Surgery. During the operation, we found that he had a horseshoe appendix with the two bases forming a circle, each communicating with the cecum. We provide a review of 13 cases presented in the literature, with a discussion of the clinical features, diagnosis, and surgical approach of the horseshoe appendix to make the general surgeon get a clear concept of this type of appendicitis.
\end{abstract}

Keywords: appendix malformation, hernia, intestinal obstruction, embryonic development, appendix neoplasm

\section{Introduction}

Many malformations of the appendix have been reported, including those involving length, location, and numbers. Duplex appendix is a rare occurrence and is reported with an incidence of $0.004 \%{ }^{1}$ Duplex appendix was systemically classified on the basis of Waugh's and Wallbridge's theory. ${ }^{2,3}$ This classification may provide some suggestions regarding the embryonic development of the cecum as well as clinical experiences.

As one type of duplex appendix, the horseshoe appendix, is extremely rare, there are only 13 reported cases (four cases in China were not recorded in English). ${ }^{4-13}$ Some cases were identified as internal hernias resulting in intestinal necrosis. ${ }^{7}$ It should be noted that two of the 13 horseshoe appendix cases involved tumors. One was a B-cell non-Hodgkin's lymphoma, and the other one was tubulovillous adenoma in the ascending colon. As the cases were very rarely reported with a high clinical risk, we advise paying closer attention to this subtype of duplex appendix taking in account that acute appendicitis is the most frequent digestive surgical emergency.

\section{Case presentation}

A 22-year-old man presented with a 3-day history of fever of $39^{\circ} \mathrm{C}$ and cough producing yellow sputum. He also appeared general malaise with nausea and vomiting. On examination, the man had rough breath sounds. An abdominal examination revealed mild epigastric tenderness without other definite positive signs, and the patient denied have had previous episodes with abdominal pain. A chest roentgenogram suggested bilateral pneumonia. His leukocyte count was elevated at $16 \times 10^{9} / \mathrm{L}$. He had a history 
of diabetes for 5 years, which was treated with insulin. He was diagnosed with pneumonia. Unexpectedly, during the 3 days of treatment with azithromycin in the Department of Pneumology, his fever never decreased. He gradually developed right lower quadrant pain with guarding and rebound tenderness. Reexamination revealed an $8 \times 8 \times 6 \mathrm{~cm}$ mass. Ultrasound showed decreased echo owing to inflammation. Abdominal computed tomography (CT) revealed gas-liquid levels and dilatation of the intestinal tract (Figure 1). The appendiceal area was obscured. According to the above examination, we judged that he already had a periappendiceal abscess at that time. The leukocyte count increased to $23 \times 10^{9} / \mathrm{L}$ at this time. Then, the patient was transferred to the Department of General Surgery with conservative treatment according to his long medical history and the formation of the appendicular abscess. We used cefoperazone sodium and sulbactam sodium combined with ornidazole. After 3 days of conservative treatment failed, surgery was performed via a right rectus abdominis incision. There was $500 \mathrm{~mL}$ of yellow-white festering around the paracolic sulcus. The tenia coli were traced down to their confluence, and the base was identified with a perforation. We decided to determine whether it was terminal, but we separated more than $10 \mathrm{~cm}$ without locating the terminus. Due to the severe adhesions, we used silk to bundle the appendix and cut it into two parts (Figure 2). Then, we separated it to a size of approximately $5 \mathrm{~cm}$ and we were surprised to find that the other base grew at the first appendix's frontal disposition. The diameter of the longer appendix was $1.0-2.0 \mathrm{~cm}$, and

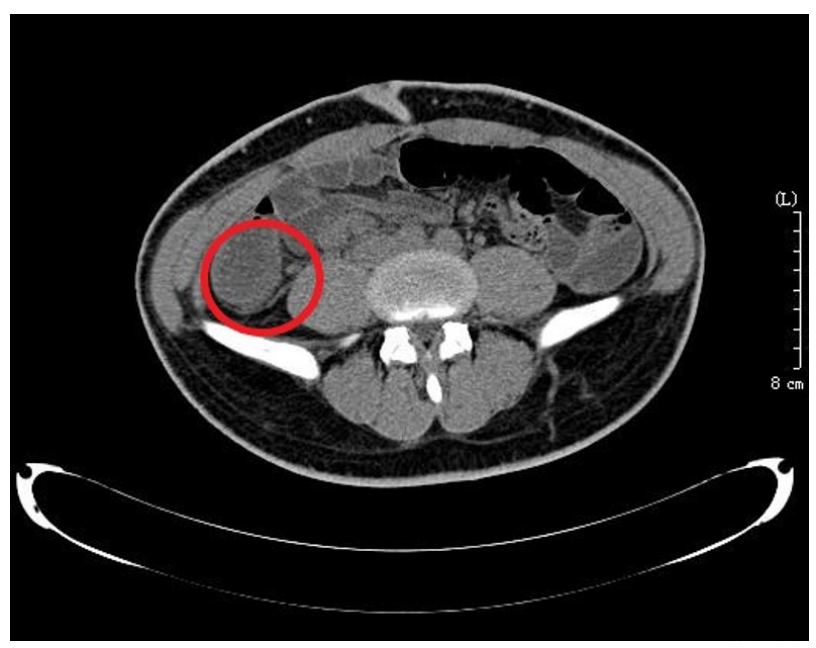

Figure I Abdominal CT showing the gas-liquid levels and dilatation of the intestinal tract and an obscured appendiceal area revealing edema of this area (circle).

Note: However, when we reviewed the CT repeatedly after the operation, the images appeared to show that the appendix was shaped like a horseshoe, a finding that was easily ignored.

Abbreviation: CT, computed tomography.

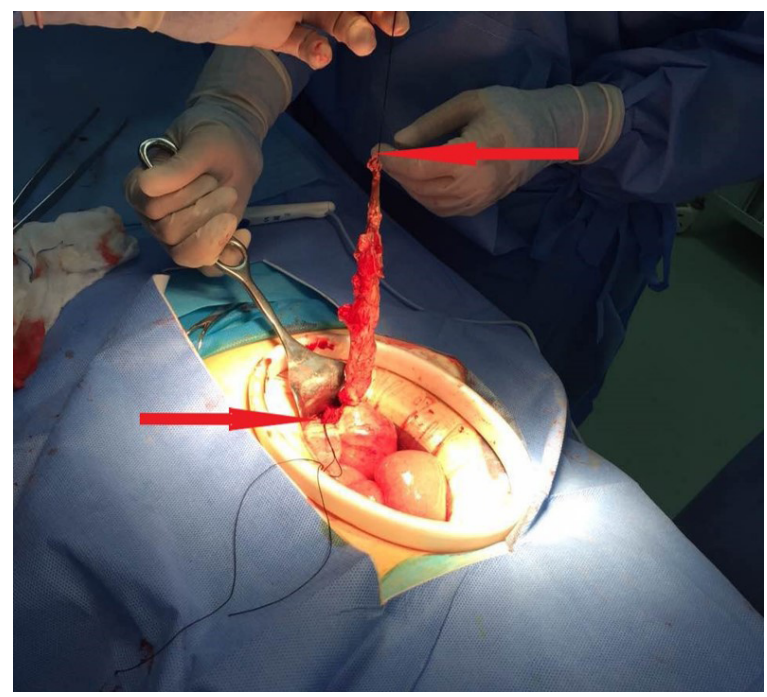

Figure 2 The horseshoe appendix was bundled and cut into two parts (arrow) to manage it side by side for severe adhesions in the appendiceal area.

that of the shorter one was $0.5-0.8 \mathrm{~cm}$. Both appendix bases were perforated, and we bundled them (Figure 3). The central mesenteric vessel was fan shaped (Figures 4 and 5). We distinguished the central mesenteric vessel from the ureter and bundled it. Both appendices were resected. The appendix was sent for pathological examination. It reported that there was marked neutrophilic infiltrated within and around the appendix. Two appendix bases were perforated and had their lumens both communicate with cecum. The postoperative recovery was uneventful except for slight hoarseness that recovered after 5 days, and the patient was discharged on postoperative day 9 .

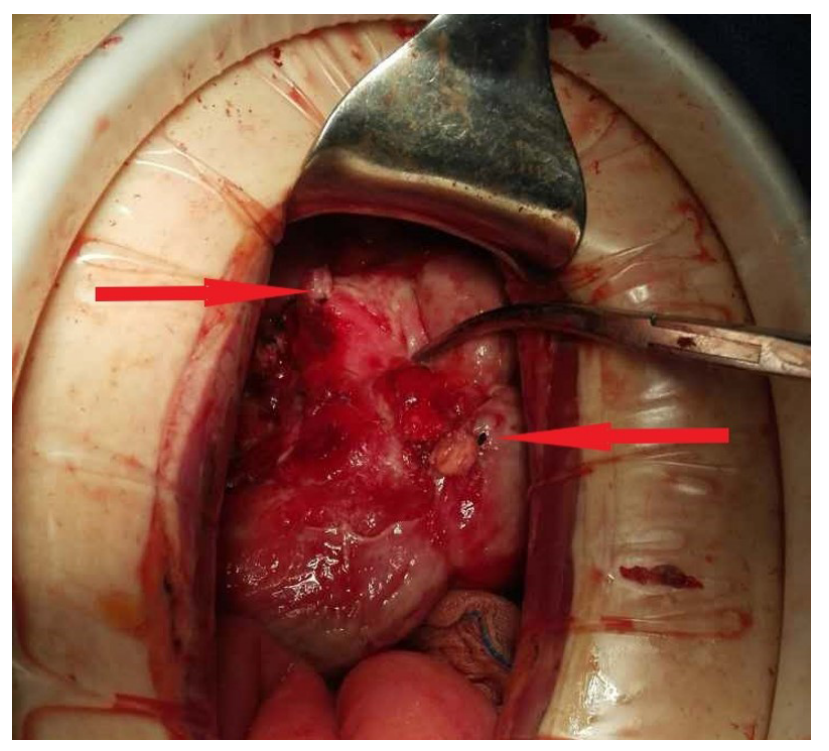

Figure 3 Both divided bases (arrow) are completely shown. 


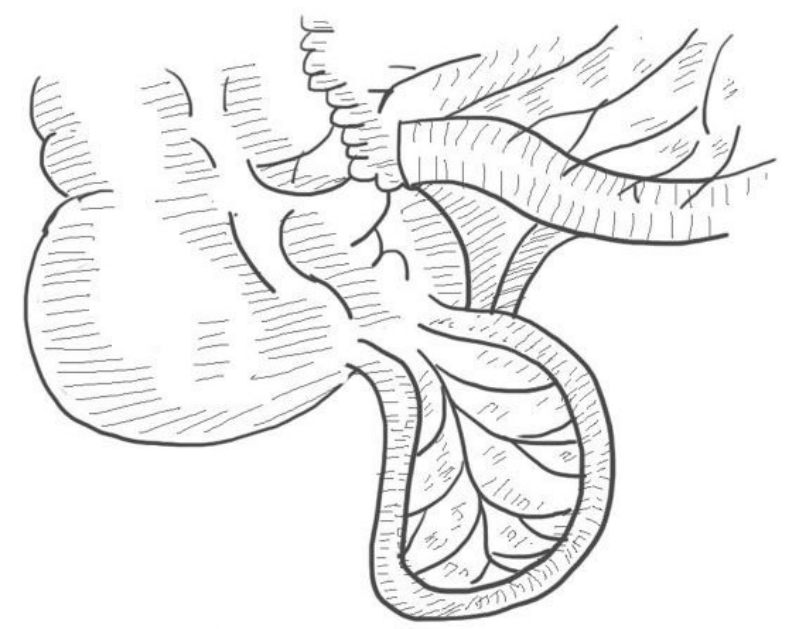

Figure 4 Frontally displaced mesentery (our case is of this type).

\section{Informed consent}

The study was approved by the Ethics Committee of The Second Affiliated Hospital of Dalian Medical University. Written informed consent was obtained from the patient to have the case details and any accompanying images published.

\section{Discussion}

A horseshoe appendix is an extremely rare type of duplex appendix. There is little information about this type of appendiceal anomaly. There are a few reports in the literature on these cases. We summarized the demographic and clinical characteristics of these patients (Table 1). ${ }^{4-13}$ Based on our review of the literature, our patient is the 14th reported case of horseshoe appendix.

Some points should be highlighted in this case:

- This case was initially diagnosed as pneumonia because he had obvious respiratory symptoms and tomography affirmed the diagnosis. However, he also had digestive

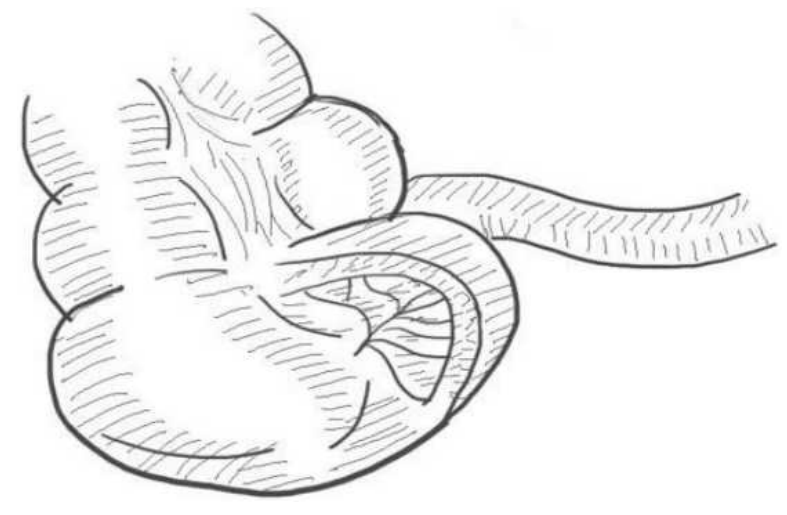

Figure 5 Sagittally displaced mesentery. tract symptoms that were easily ignored because patients with pneumonia often have general malaise with fever. Nevertheless, we should consider evaluating whether patients have other diseases to avoid delaying diagnosis and avoiding risk.

- We scanned his abdomen with ultrasound and CT before surgery and were unable to diagnose horseshoe appendix before surgery. However, we reviewed the CT repeatedly after surgery and the images appeared to show that the appendix was shaped like a circle (Figure 1), a finding that was easily ignored.

- Although we used a large incision, we did not find other organ malformations.

We analyzed the 14 reported cases (Table 1), including nine men and five women who ranged in age from 4 to 78 years (with an average age of 41 years). Patients were diagnosed with appendicitis (7/14), appendicular mass (2/14), bowel occlusion (3/14), B-cell non-Hodgkin's lymphoma (1/14), and tubulovillous adenoma of the ascending colon (1/14). Horseshoe appendix is difficult to diagnose preoperatively; therefore, ultrasound and three-dimensional reconstruction may be helpful. ${ }^{5}$ Only two patients were diagnosed with horseshoe appendix preoperatively by ultrasound and three-dimensional rebuilt images. ${ }^{5}$

There is a clear indication for surgery in symptomatic cases. Except for one case diagnosed with B-cell nonHodgkin's lymphoma treated with chemotherapy in addition to simple resection, ${ }^{11}$ the other appendices were simply removed. The treatment of choice is complete surgical excision. In asymptomatic cases, whether they should be treated conservatively or not requires close follow-up.

We found the appendix to be supplied by a single fanshaped vessel, which was confirmed by other cases. The patients can be classified into the following two types based on the location of the mesentery: frontal types (6/14), with the bases of the appendix not on the tenia (Figure 4), and sagittal types (5/14), with the bases of the appendix along the tenia (Figure 5). As for the orifice of the bases, almost all cases showed that the appendix communicated with the cecum at both ends, except for two cases: one that showed communication from the cecum to the ascending colon ${ }^{5}$ and the other one that showed communication from the cecum to the hepatic flexure of the colon. ${ }^{6}$

A feature that made us pay more attention to this type of appendiceal malformation was revealed by Drino et al, ${ }^{7}$ who described a horseshoe appendix that induced an internal hernia, resulting in intestinal necrosis (3/14). Their patient's postoperative course was complicated by partial gangrene of 


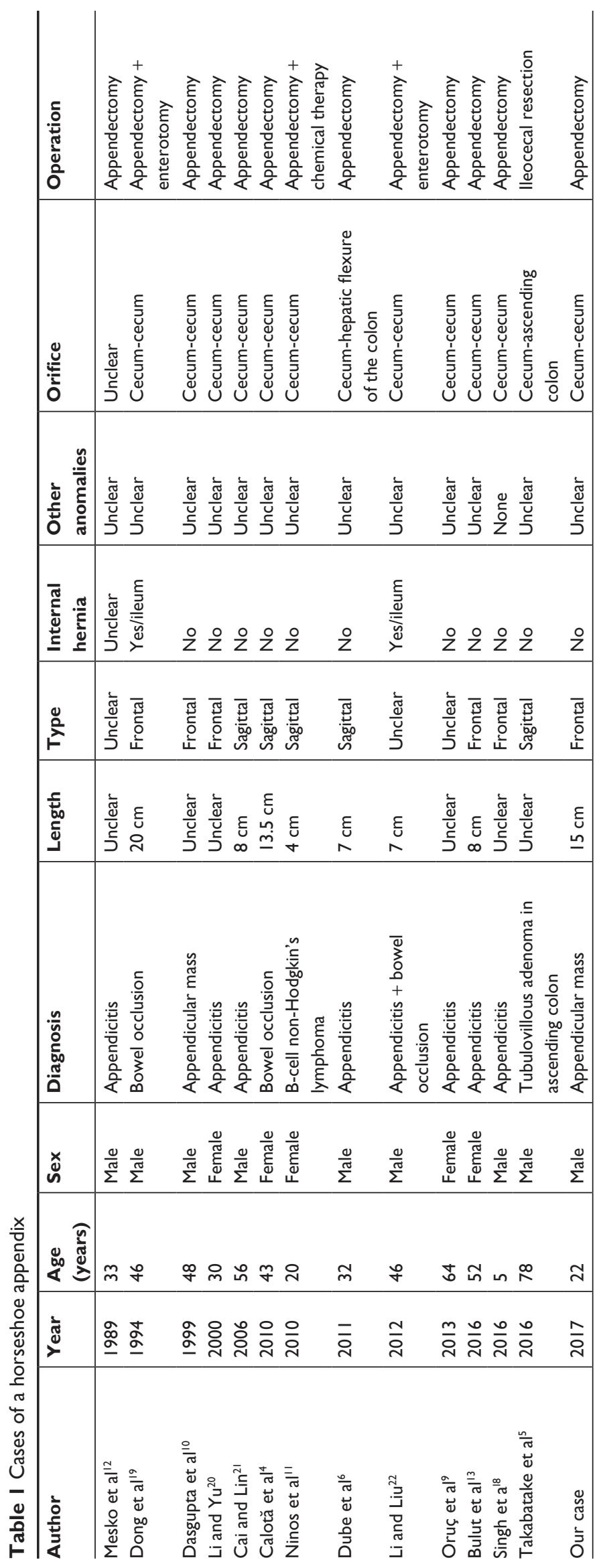


the cecum and fistula of the intestines on a completely intact part of the intestinal wall, possibly explained by the presence of anomalous vascularization in the area. ${ }^{7}$ This anomalous vascularization was also found in two cases reported by Chinese specialists. In addition, we should pay more attention to whether patients have other malformations. According to some reports, when a duplication of appendix is encountered in these patients, other intestinal, genitourinary, or vertebral malformations must be explored, requiring our attention. The published case reports were deficient in terms of this information. Whether these malformations have a relationship with tumorigenesis needs to be explored. In only one other case, there was a search for other malformations during the operation, ${ }^{8}$ which should be emphasized in future studies.

Some classifications of appendiceal anomalies have been developed by Cave ${ }^{14}$ and Biermann et al. ${ }^{15}$ In 2010, Calotă et $\mathrm{al}^{4}$ reported a classification system for anomalies of the appendix, which were modified by Takabatake et $\mathrm{al}^{5}$ to cover the types of horseshoe appendix. The classification of appendiceal anomalies is provided in Table 2. ${ }^{2-5,14,15}$

Duplication of the appendix ranges from branching of the appendiceal trunks to a fully mature appendix located elsewhere along the colon, as described by Nageswaran et $\mathrm{al}^{18}$ in the beginning of 2018. Most duplications of the appendix can be categorized according to the Cave-Wallbridge classification. There are 22 Type A, eight Type B1, 46 Type B2, and 10 Type $\mathrm{C}$ cases (Cave-Wallbridge) reported. There are 13 cases of horseshoe appendix (including four cases in China) and two cases of triple appendix reported.

Based on the classification of the horseshoe appendix, some experts have tried to provide embryological explanations for its development. The appendix is a diverticulum of the cecum. The appendix increases excessively in length with the right wall of the cecum pushing the appendix down immediately. ${ }^{16}$ There are also four theories for gastrointestinal duplication: the split notochord theory; median septum formation; normal regression of embryonic diverticula; and partial twinning procedure ${ }^{17}$ One phenomenon was described by Kelly, Hurden, Gladstone, and Wakeley in which a transient appendix develops from the terminus of the cecum at the initial fifth week and atrophies at the seventh week and soon disappears. The remaining appendix continues to grow. Cave ${ }^{14}$ believes that the transient appendix may be substantiation of the duplication of the appendix. As a type of duplex appendix, however, there is no definite proof of how a horseshoe appendix develops.

Table 2 Classification of appendiceal anomalies

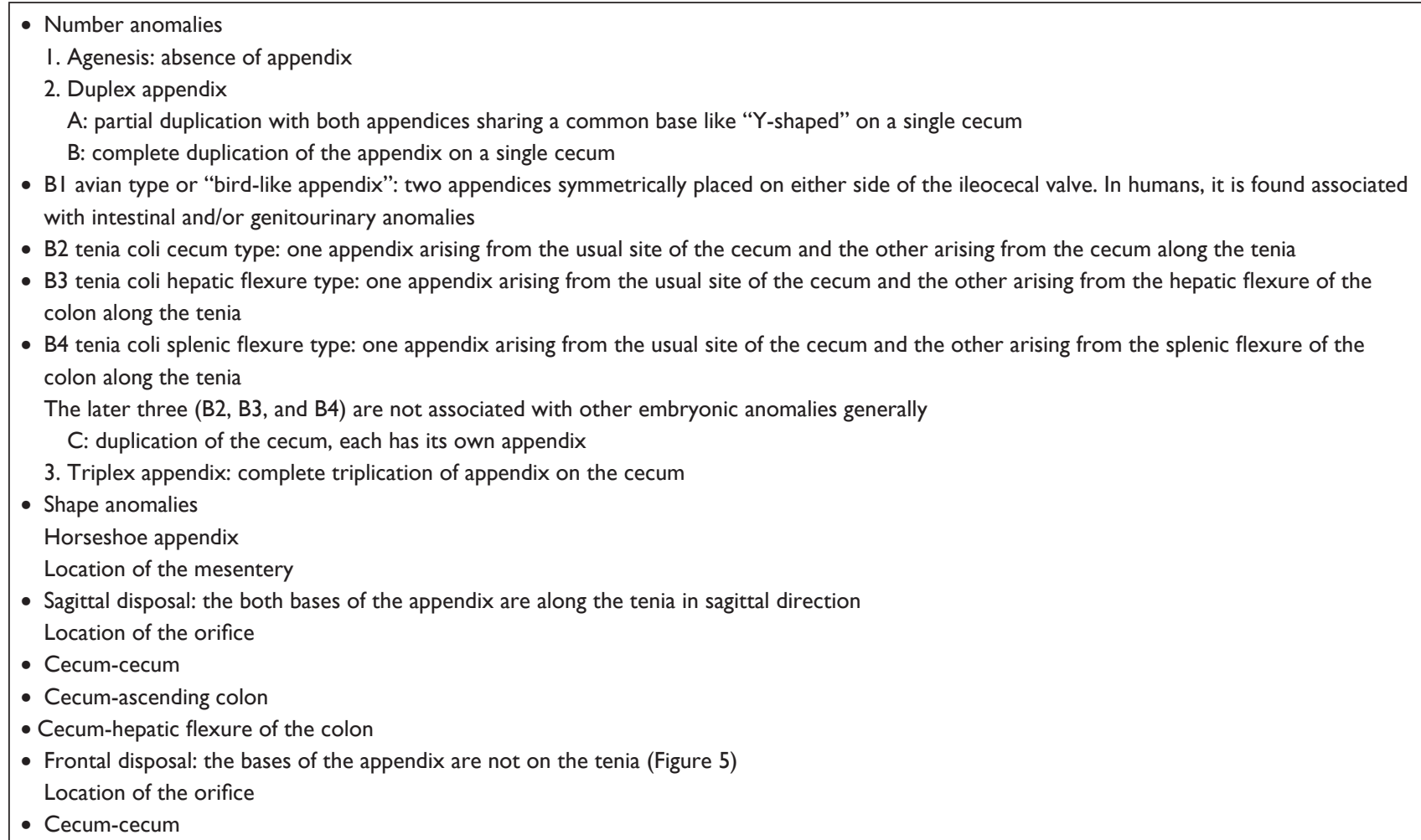

Note: Data from these studies. ${ }^{2-5,14,15}$ 


\section{Conclusion}

Surgeons should consider this abnormal structure, even though it is rare. If a malformation of the appendix is found which is difficult to manage, the incision should be changed in a timely fashion, instead of insisting on the McBurney incision. When the behavior of the appendix does not correspond to the symptoms or the diagnosis of the patients, especially in patients who have had previous surgery for congenital abnormalities, a Type B1 or Type C duplication should be considered. Similarly, for an anteriorly placed appendix, away from the convergence of the tenia, it should be determined whether there are other anatomic malformations. A careful examination of the cecal pole should be performed, and the retrocecal space should be explored for appendiceal malformations. More case reports of horseshoe appendices are needed, and it should be determined whether horseshoe appendix is related to tumorigenesis.

\section{Acknowledgments}

The authors would like to thank the participant and staff of the Center for the New Material and New Precision Technology of Cancer Therapy, Dalian Medical University, and the Division of Hepatobiliary and Pancreatic Surgery, Department of General Surgery, The Second Affiliated Hospital of Dalian Medical University, for their valuable contributions. This work was supported by the fund from the National Natural Science Foundation of China (81471755).

\section{Author contributions}

Jin Liu, Chengyong Dong, and Liming Wang developed the main concept and designed the study. All of the authors participated in the management of the patient in this case report. Jin Liu performed the literature review and drafted the manuscript. Chengyong Dong, Haibo Wang, Zhenming Gao, Rui Liang, and Deguang Sun supervised the case and also helped edit the manuscript. Liming Wang contributed to the editing and critical revision for important intellectual content. All authors contributed toward data analysis, drafting and critically revising the paper and agree to be accountable for all aspects of the work.

\section{Disclosure}

The authors report no conflicts of interest in this work.

\section{References}

1. Kjossev KT, Losanoff JE. Duplicated vermiform appendix. Br J Surg. 1996;83(9): 1259.

2. Waugh TR. Appendix vermiformis duplex. Arch Surg. 1941;42(2): 311-320.

3. Wallbridge PH. Double appendix. Br J Surg. 1962;50(221): 346-347.

4. Calotă F, Vasile I, Mogoantă S, et al. Horseshoe appendix: a extremely rare anomaly. Chirurgia. 2010;105(2):271-274.

5. Takabatake K, Ikeda J, Furuke H, et al. A case of a horseshoe appendix. Surg Case Rep. 2016;2(1):140.

6. Dube B, Manoharan GR, Daya M. Anomalous origin of the vermiform appendix. S Afr J Surg. 2011;49:100.

7. Drino E, Radnić D, Kotjelnikov B, Aksamija G. Rare anomalies in the development of the appendix. Acta Chir Iugosl. 1991;38(1):103-111.

8. Singh C, Nyuwi KT, Rangaswamy R, Ezung YS, Singh HM. Horseshoe Appendix: An Extremely Rare Appendiceal Anomaly. J Clin Diagn Res. 2016;10(3):25-26.

9. Oruç C, Işı1k O, Ureyen O, Kahyaoğlu OS, Köseoğlu A. An extremely rare appendiceal anomaly: horseshoe appendicitis. Ulus Travma Acil Cerrahi Derg. 2013;19(4):385-386.

10. Dasgupta R, Reber PU, Patel AG. Horseshoe appendicitis. Eur J Surg. 1999;165(11):1095-1096.

11. Ninos A, Douridas G, Papakonstantinou E, et al. A horseshoe double appendix positioned on a non-Hodgkin lymphoma. Hellenic Journal of Surgery. 2010;82(1):73-76.

12. Mesko TW, Lugo R, Breitholtz T. Horseshoe anomaly of the appendix: a previously undescribed entity. Surgery. 1989;106(3):563-566.

13. Bulut SP, Cabığlu N, Akıncı M. Perforated double appendicitis: Horseshoe type. Ulus Cerrahi Derg. 2016;32(2):134-136.

14. Cave AJ. Appendix Vermiformis Duplex. J Anat. 1936;70(Pt 2): 283-292.

15. Biermann R, Borský D, Gogora M. Double appendicitis - a rare pathologic entity. Chirurg. 1993;64(12):1059-1061.

16. Malina RM. The developing human: Clinically oriented embryology. Am J Hum Biol. 1993;5(4):507.

17. Jimenez SG, Oliver MR, Stokes KB, Morreau PN, Chow CW. Case report: Colonic duplication: a rare cause of obstruction. J Gastroenterol Hepatol. 1999;14(9):889-892.

18. Nageswaran H, Khan U, Hill F, Maw A. Appendiceal Duplication: A Comprehensive Review of Published Cases and Clinical Recommendations. World J Surg. 2018;42(2):574-581.

19. Dong Z, Fu X, Luo H. [Horseshoe appendix induced intestinal obstructon]. Chinese J Clinical Anatomy.1994;12(4):309-310. Chinese.

20. Li C, Yu J. [A horseshoe appendix]. Guangdong Med J. 2000;21(11):982983. Chinese.

21. Cai S, Lin M. [A case of annular appendix with acute appendicitis]. $J$ Med Imaging. 2017;17;(1):27-28. Chinese.

22. Liu J, Li D. [man xing huan zhuang lan wei yan ji xing fa zuo bing qian dun xing hui chang nei zuo yi li]. Chin J Gastrointest Surg. 2012;15(8):786-787. Chinese.
Therapeutics and Clinical Risk Management

\section{Publish your work in this journal}

Therapeutics and Clinical Risk Management is an international, peerreviewed journal of clinical therapeutics and risk management, focusing on concise rapid reporting of clinical studies in all therapeutic areas, outcomes, safety, and programs for the effective, safe, and sustained use of medicines. This journal is indexed on PubMed Central, CAS,

\section{Dovepress}

EMBase, Scopus and the Elsevier Bibliographic databases. The manuscript management system is completely online and includes a very quick and fair peer-review system, which is all easy to use. Visit $\mathrm{http}: / / \mathrm{www}$.dovepress.com/testimonials.php to read real quotes from published authors. 